\title{
Geometry-controlled kinetics
}

\author{
O. Bénichou, ${ }^{1}$ C. Chevalier,${ }^{1}$ J. Klafter,${ }^{2}$ B. Meyer, ${ }^{1}$ and R. Voituriez ${ }^{1}$ \\ ${ }^{1}$ UPMC Univ Paris 06, CNRS-UMR 7600 Laboratoire \\ de Physique Théorique de la Matière Condensée, \\ 4 Place Jussieu, F-75005 Paris, France. \\ ${ }^{2}$ School of Chemistry, Raymond and Beverly Sackler Faculty of Exact Sciences, \\ Tel Aviv University, Tel Aviv, Israel; and Institute for Advanced Studies (FRIAS), \\ University of Freiburg, 79104, Freiburg, Germany.
}

(Dated: June 1, 2018)

\begin{abstract}
It has long been appreciated that transport properties can control reaction kinetics. This effect can be characterized by the time it takes a diffusing molecule to reach a target - the first-passage time (FPT). Although essential to quantify the kinetics of reactions on all time scales, determining the FPT distribution was deemed so far intractable. Here, we calculate analytically this FPT distribution and show that transport processes as various as regular diffusion, anomalous diffusion, diffusion in disordered media and in fractals fall into the same universality classes. Beyond this theoretical aspect, this result changes the views on standard reaction kinetics. More precisely, we argue that geometry can become a key parameter so far ignored in this context, and introduce the concept of "geometry-controlled kinetics". These findings could help understand the crucial role of spatial organization of genes in transcription kinetics, and more generally the impact of geometry on diffusion-limited reactions.
\end{abstract}

PACS numbers: 
It is known on general grounds that reaction kinetics can be influenced by the transport properties of the reactants [1-3]. Actually, the transport step, before reactants meet and eventually react, even becomes limiting in the case of confined systems such as cells or cell subdomains where a small number of reactants are involved [4-9]. In such systems, the first step in estimating the kinetics of reactions consists in evaluating the properties of firstencounter between reactants. Quantitatively, this amounts to calculating the distribution of the time it takes a diffusing molecule to reach a target site - the first-passage time (FPT) distribution. While this quantity is well-known in quasi-one dimensional or unconfined geometries (see [10] for a review), determining the FPT distribution appears to be intractable in the realistic situation where the diffusing molecule is confined within a finite domain [11. A first estimate of the effect of geometrical parameters of confinement on this search time is given by the mean of the FPT. This has recently been calculated, and a linear scaling with the volume has been demonstrated [12 15]. However, as soon as several time scales are involved, the kinetics can not be determined by the mean of the FPT only, and the entire distribution is needed [16, 17].

Gene transcription provides an extremely important example - which we shall repeatedly invoke in what follows - of reactions involving a small copy number of (or even single) reactants confined within a small (micron-sized) domain, and whose kinetics must be precisely regulated to fulfill vital cell functions. Interest in the question of how geometrical parameters impact on the kinetics of such transcriptional reactions and could act as regulatory factors has recently increased, mainly because of new experimental tools that enable the observation of the real-time production of proteins at the single molecule level [4]. These technics, which give access to the spatial organization of the genetic material, have revealed strong correlations between the spatial locations of successively activated genes, both for prokaryotes [18] and eukaryotes [19]. Indeed, it has been found that successively activated genes are often colocalized, that is located in the very same nuclear regions. These observations raise the question of the importance of geometrical parameters in transcription kinetics, which has remained so far widely unanswered.

In the broader context of chemical reactions in confinement, we are interested here in the following questions : (i) How does the FPT distribution depend on the volume of the confining domain? (ii) How does it depend on the initial position of the diffusing molecule, and (iii) is this geometric dependence an important factor, which could potentially control 
the kinetics?

Note that the influence of confinement and crowding effects on biochemical reactions has already been studied (see [20, 21] for reviews) on the basis of a thermodynamical treatment of reaction kinetics. While this approach is well suited to the case of a large number of reactants, it does not provide the dependence of the kinetics on the geometrical parameters mentioned above (such as the initial position of the reactants), which involve the individual nature of the reactants and their dynamical properties.

In this work, we calculate analytically the distribution of the FPT at a target $T$ for a diffusing particle released at a starting point $S$ (see figure 1) and quantitatively answer questions (i)-(iii) above. We highlight universal laws of the FPT distribution as function of the volume $N$ of the confining domain and of the distance $S T \equiv r$ and show that two regimes emerge. More precisely, we find that the key criterion is the compact vs non compact nature of the diffusion process, to be defined mathematically below. In the non compact case, which physically corresponds to a diffusing molecule which "sparsely" explores its environment and leaves unvisited regions (typically a molecule diffusing in a dilute solution), we show that the kinetics is widely independent of the starting point. On the opposite compact case of a diffusing molecule which "densely" explores its environment (for example a molecule in a very crowded medium), the position of the starting point strongly influences the search time of the target, which leads us to introduce the concept of "geometry-controlled kinetics". In the context of gene transcription, this result implies that the kinetics of activation of a gene $T$ by a transcription factor $(\mathrm{TF})$ can be orders of magnitudes faster if the $\mathrm{TF}$ is released from a site which is colocalized with (i.e. in the vicinity of) $T$, as compared to the case where the $\mathrm{TF}$ is released from a remote site.

\section{Results}

We consider a Markovian random walker of position $\mathbf{r}(t)$, whose dynamics is characterized by the dimension of the walk $d_{w}$, defined by the scaling of the mean squared displacement $\left\langle\mathbf{r}^{2}(t)\right\rangle \propto t^{2 / d_{w}}$. The walker is confined in a domain of $N$ with reflecting walls. Additionally, we assume that the medium is of fractal type, so that its characteristic linear size $R$ scales as $R \propto N^{1 / d_{f}}$, where $d_{f}$ is the fractal dimension [22]. We are interested in the distribution $P\left(\mathbf{T}_{T S}\right)$ of the time it takes a walker starting from the site $S$ to reach for the first time the 
target site $T$ located at a distance $r$ from $S$.

We start from the backward equation satisfied by the probability $P\left(\mathbf{T}_{T S}=t\right)$ in discrete time $t$ [10]

$$
P\left(\mathbf{T}_{T S}=t\right)=\sum_{j} w_{j S} P\left(\mathbf{T}_{T j}=t-1\right),
$$

obtained by partitioning over the first step of the walk, where $w_{i j}$ stands for the transition probability from site $j$ to site $i$. It is shown in Supplementary Information (SI) that this equation, after Laplace transform, leads to the following hierarchy satisfied by the moments $\left\langle\mathbf{T}_{T j}^{n}\right\rangle$ of the FPT :

$$
\left\langle\mathbf{T}_{T S}^{n}\right\rangle=\frac{1}{W_{T}^{\text {stat }}} \sum_{j} \sum_{k=1}^{n}\left(\begin{array}{l}
n \\
k
\end{array}\right)(-1)^{k+1}\left[\left(H_{T T}-H_{T S}\right) W_{j}^{\text {stat }}+\left(H_{j S}-H_{j T}\right) W_{T}^{\text {stat }}\right]\left\langle\mathbf{T}_{T j}^{n-k}\right\rangle .
$$

Here

$$
H_{j i}=\sum_{n=0}^{\infty}\left(W_{j i}(n)-W_{j}^{\text {stat }}\right)
$$

and $W_{j i}(n)$ denotes the propagator of the walk, ie the probability to be at site $j$ at step $n$ starting from site $i$, while $W_{j}^{\text {stat }}$ is the probability to be at site $j$ in the stationary state.

At this stage, the hierarchy of equations (2) remains formal, as it involves the unknown functions $H_{j i}$, and does not allow an explicit determination of the FPT distribution. However, this difficulty can be circumvented by taking the large volume limit and by considering the rescaled time $\theta=\mathbf{T}_{T S} / \overline{\langle\mathbf{T}}_{T}$, where $\overline{\langle\mathbf{T}}_{T}=\sum_{S}\left\langle\mathbf{T}_{T S}\right\rangle W_{S}^{\text {stat }}$ stands for the mean FPT to the target site $\mathbf{r}_{T}$, averaged over the initial position. Note that here we implicitly assume that $\overline{\langle\mathbf{T}}_{T}$ (as well as its disorder average in the case of disordered systems to be discussed below) is finite. Actually, a detailed analysis of Eq.(2) allows us to show in SI that the distribution of the rescaled variable $\theta$ takes in the large volume limit the following general form

$$
G_{T S}(\theta)=\left(1-\Pi_{T S}\right) \delta(\theta)+\Pi_{T S} \psi(\theta)
$$

where the Dirac $\delta$ function corresponds to trajectories hitting the target without reaching the boundary within a time of order $r^{d_{w}}$ much smaller than $\overline{\langle\mathbf{T}\rangle_{T}}$. The geometrical factor $1-\Pi_{T S}$ can be interpreted as the weight of these trajectories. Similarly, the contribution $\Pi_{T S} \psi(\theta)$ accounts for trajectories reaching the boundary before the target. Note that the dependence on the starting point lies entirely in the geometrical factor $\Pi_{T S}$, whereas the 
time dependence is contained in the scaling function $\psi$. The geometrical factor $\Pi_{T S}$ and the rescaled variable $\theta$ are explicitly determined in SI and their scaling with the volume $N$ and the distance $r$ are obtained under the standard scale-invariance assumption of the unconfined propagator $W_{i j}^{\infty}(n) \propto n^{-d_{f} / d_{w}} f\left(\left|\mathbf{r}_{i}-\mathbf{r}_{j}\right| / n^{1 / d_{w}}\right)[22$. Actually, the FPT distribution given by Eq. (4) falls into a few universality classes, defined according to a purely geometrical criterion as detailed below.

In the case of non compact exploration defined here by $d_{w}<d_{f}$ [23], where the mean number of distinct sites visited by the walker in absence of confinement grows linearly with the number of steps, we find :

$$
\left\{\begin{array}{l}
{\overline{\langle\mathbf{T}\rangle_{T}}}=H_{T T} / W_{T}^{\text {stat }} \propto N \\
\Pi_{T S}=\frac{\left\langle\mathbf{T}_{T S}\right\rangle}{\overline{\langle\mathbf{T}\rangle_{T}}} \propto 1-\alpha\left(\frac{1}{r}\right)^{d_{f}-d_{w}} \\
\psi(\theta)=e^{-\theta}
\end{array}\right.
$$

where $\alpha$ is a lattice dependent constant of order 1 . Note that the linear dependence on $N$ of the scaling variable $\overline{\langle\mathbf{T}}_{T}$ is the same as found in [13] for the mean $\mathrm{FPT}\left\langle\mathbf{T}_{T S}\right\rangle$, and in particular does not depend of the dimensions $d_{f}$ and $d_{w}$. This result includes as a special case regular diffusion in 3D given in [24] for which $d_{w}=2$ and $d_{f}=3$. Strikingly, the exponential form of $\psi$ holds for any dimensions such that $d_{w}<d_{f}$. Note that while the FPT distribution is a mere exponential of weight one in the limit of $r$ larger than the step size, it departs significantly from this distribution if the starting point is close to the target.

In the opposite case of compact exploration $d_{w}>d_{f}$ [23], where the mean number of distinct sites visited by the walker in absence of confinement grows slower than linearly with the number of steps, further hypothesis on the unconfined propagator are needed to estimate the relative importance of the terms involved in Eq. (2). Making use of the O'ShaughnessyProcaccia operator [25] to evaluate the large volume behaviour of $H_{i j}$ in (2), we find that the FPT distribution obeys the generic form of Eq. (4) with

$$
\left\{\begin{array}{l}
{\overline{\langle\mathbf{T}\rangle_{T}}}=H_{T T} / W_{T}^{\text {stat }} \propto N^{d_{w} / d_{f}} \\
\Pi_{T S}=\frac{2 d_{f}^{2}}{d_{w}\left(d_{f}+d_{w}\right)} \frac{\left\langle\mathbf{T}_{T S}\right\rangle}{\overline{\langle\mathbf{T}\rangle_{T}}} \propto\left(\frac{r}{R}\right)^{d_{w}-d_{f}} \\
\psi(\theta)=\frac{2 d_{f} d_{w}}{d_{w}^{2}-d_{f}^{2}} \frac{\Gamma(\nu)}{\Gamma(2-\nu)} \sum_{k=0}^{\infty} \frac{\left(\frac{\alpha_{k}}{2}\right)^{3-2 \nu} J_{\nu}\left(\alpha_{k}\right)}{J_{1-\nu}\left(\alpha_{k}\right)} e^{-\frac{\alpha_{k}^{2} d_{w} d_{f}}{2\left(d_{w}^{2}-d_{f}^{2}\right)} \theta}
\end{array}\right.
$$

where $\theta>0, \nu=d_{f} / d_{w}$ and $\alpha_{0}<\alpha_{1}<\ldots$ stand for the zeros of the Bessel function $J_{-\nu}$. Strikingly, the scaling with $N$ of the scaling variable $\theta$ is no longer given by the mean FPT 
$\left\langle\mathbf{T}_{T S}\right\rangle$, which clearly indicates that several time scales are involved in the problem. The interplay between these time scales leads to a non trivial family of universal non exponential scaling functions, parametrized by $d_{w}$ and $d_{f}$. The geometrical factor strongly depends on the source-target distance $r$, and in particular is very small for $r \ll R$, as opposed to the non compact situation. We add that the marginal case $d_{w}=d_{f}$, which is compact according to the definition given in [23], corresponds to an exponential scaling function $\psi$ as given by Eq. (5), with logarithmic corrections in the scalings of $\overline{\langle\mathbf{T}\rangle}\rangle_{T}$ and $\Pi_{T S}$ with $r$ and $N$ (see SI).

Eqs(5)-(6) fully define universality classes of FPT distributions in confinement. Additional comments are in order: (i) Whereas the linear scaling with $N$ of the first moment is universal, the scaling of higher moments differ in compact and non compact cases. In particular, this scaling implies that while the reduced variance of the FPT is always of order one in the non compact case, in the compact case it reads $\left(\left\langle\mathbf{T}_{T S}^{2}\right\rangle-\left\langle\mathbf{T}_{T S}\right\rangle^{2}\right) /\left\langle\mathbf{T}_{T S}\right\rangle^{2} \propto(R / r)^{d_{w}-d_{f}}$, so very large fluctuations occur for $r \ll R$. (ii) Remarkably, the FPT distribution is entirely determined as soon as the mean $\left\langle\mathbf{T}_{T S}\right\rangle$ of the FPT is known (as well as its average over the starting point $\overline{\langle\mathbf{T}}_{T}[26-30]$ ), even if the distribution is not exponential. (iii) For specific cases, this mean FPT can be calculated exactly, which provides a fully explicit expression of the FPT distribution (as used in fig 2.b, 3.a, 3.b) (iv) In all cases it can be calculated in the large volume limit using the recent result [13], leading to the scaling in the geometrical parameters given in Eqs(5)-(6) .

We note that our approach covers in particular the important case of subdiffusion [31, which is characterized by a sublinear dependence of the mean squared displacement with time (that is $d_{w}>2$ ). Subdiffusion is widespread in complex crowded environments such as biological cells [32, 33], and might physically originate from a few classes of models based on different underlying microscopic mechanisms [34]. Importantly, subdiffusive processes can be either compact or non-compact, which will prove below to be the relevant criterion in the context of reaction kinetics. The FPT distribution for one class of models for subdiffusion, which rely on spatial inhomogeneities [22] as exemplified by diffusion in fractals, is directly given by Eqs.(5)-(6). Another class of models stems from large trapping times, leading to the case of infinite $\overline{\langle\mathbf{T}}_{T}$, which we have discarded so far. While the quenched version of this type of model becomes quite involved in the case of broad distribution of trapping times over the disorder, the FPT distribution in the annealed case - the continuous time random walk model (CTRW), which is a standard random walk with random waiting times, drawn 
from a distribution $f(t)$ [31, 35-37] - is straightforwardly deduced from Eqs.(5)-(6). In this case, the Laplace transform of the FPT distribution reads: $\widehat{P}_{\mathrm{CTRW}}(s)=\widehat{P}(\widehat{f}(s))$, where $\widehat{P}(s)$ is the generating function (discrete Laplace transform) of the FPT distribution of the underlying discrete time random walk, which is determined in Eqs.(5)-(6).

These analytical results are validated by Monte Carlo simulations and exact enumeration methods applied to various models which illustrate the universality classes defined above. These schematic models have been widely used to describe transport in disordered media [16, 22], for example in the case of exciton trapping on percolation systems [38] or anomalous diffusion in biological cells [39, 40], as a first step to account for geometrical obstruction and binding effects involved in real crowded environments [20, 21]. (i) The non compact and marginal cases (see fig 2) are exemplified by regular diffusion on a 3D and 2D cubic lattices, diffusion on a 3D percolation cluster above criticality, and diffusion in disordered systems such as the random barrier model (namely a symmetric random walk on a 3D cubic lattice with transition rates $\Gamma$ drawn from the normalized distribution $\left.\rho(\Gamma) \propto \Gamma^{-\alpha}\right)$ and the random trap model (namely a symmetric random walk on a 3D cubic lattice with frozen waiting times $\tau_{i}$ at each site drawn from the normalized distribution $\rho(\tau) \propto \tau^{-(1+\alpha)}$ ) . (ii) The compact case (see fig 3) is exemplified by diffusion on deterministic fractals such as Sierpinski gasket and T-graph and on a critical percolation cluster, as defined in fig 1 of the SI. Figures 2 and 3 reveal an excellent quantitative agreement between the asymptotic analytical predictions and the numerical simulations, even for systems of small size. We emphasize that despite their very different nature, all these models fall into the above defined universality classes.

\section{Discussion}

We now discuss important implications of these results for reaction kinetics, using the ubiquitous example of a target search process involving an immobile target $B$ and searcher particles $A$ [1]. When only a small number of $A$ are involved in the reaction, as is the case in a microdomain in a biological cell, this reaction has to be described at the single molecule level [6] and can be quantified by the survival probability of a particle $A, S(t)=1-\sum_{t^{\prime}=0}^{t} P\left(t^{\prime}\right)$, which gives the probability that $A$ has not reacted with $B$ until time $t$. The quantity $S(t)$ depends on the initial position of the molecule $A$ and is explicitly determined using Eqs (5)(6), which indicate that such reactions in confinement obey universal kinetic laws, depending 
on the non compact vs compact nature of the underlying transport process:

(i) In the non compact case, which corresponds qualitatively to trajectories leaving many sites unvisited, as in the case of a 3D medium dilute enough to lead to regular diffusion, for any $r$ significantly greater than a typical molecular length, the geometrical factor $\Pi_{T S}$ is close to 1, and the dependence on the initial position is lost. We therefore recover a simple first order decay of the survival probability which depends on the volume of the confining domain only, and not on the initial position of the reactant. In this case of non compact exploration, the initial position is not an important parameter of the kinetics (see fig. 4), except in specific cases involving return times, such as recombination reactions .

(ii) On the contrary, in the compact case, which means that each visited site is on average oversampled, geometrical factors dominate. This is typically the case of a crowded medium described to a first approximation as a fractal structure where the available space for diffusion is restricted. Here, the temporal evolution of $S(t)$ strongly depends on the starting position. $S(t)$ drops to small values, indicating that the reaction occurred with high probability, on a time scale which depends on the volume, but also critically on the starting position of the reactant. This time scale ranges according to $\mathrm{Eq}(4)$, (6) from $r^{d_{w}}$ (for starting positions such that $r \ll R$ ) to $R^{d_{w}}$ (for starting positions such that $r \simeq R$ ), which in practice can span several orders of magnitude (see fig. 4). In this type of "geometry-controlled reactions" (not to be confused with "fractal-like reactions" [2]), spatial organization of reactants plays a crucial role, which can be quantified by our approach.

We stress that the decisive criterion leading to geometry controlled kinetics is not the subdiffusive $v s$ diffusive nature of the transport process, but its compact $v s$ non compact type. We expect this effect to impact a wide class of reactions involving either an inhomogeneous initial concentration of reactants, such as a speckeled distribution as experimentally realized in [41, or a small number of particles, such as biochemical reactions in cell subdomains. Notably, in the context of gene colocalization, our results give access to the kinetics of elementary steps of activation by transcription factors. As an illustrative example, let us consider two genes $A$ and $B$ which share a common transcription factor (TF) (for example the genes sog and zen of the Drosophila genome which are both targeted by Dorsal [42]). Experimental results concerning subdiffusive motion of tracer particles in the nucleus [32, 43, 44] on the one hand and observations of a fractal organization of the chromatin on the other hand [44-46], provide the following estimates $2 \leq d_{w} \leq 3$ and $d_{f} \simeq 2.4$, and therefore 
suggest that both compact and non compact exploration cases could occur. Relying on the analysis of the survival probability developed previously, we find in the case of compact exploration (with for example $d_{f}=2.4$, and $d_{w}=3$ as in [43]) that the typical time needed for the TF to reach gene $B$ starting from a gene $A$ colocalized with, i.e. in the vicinity of, $B$ (typically $r_{A B}=r_{\text {coloc }} \leq 100 \mathrm{~nm}$, which is the size of a transcription factory [19]) can

be $\left(r_{\text {remote }} / r_{\text {coloc }}\right)^{d_{w}} \simeq 10^{3}$ times faster than for a remote gene $A$ (typically $r_{A B}=r_{\text {remote }} \simeq$ $1 \mu \mathrm{m}$, which is the order of magnitude of a nucleus radius). This is in strong contrast with the case of non compact exploration (with for example $d_{f}=2.4$, and $d_{w}=2$ [32]) where the typical activation time of B starting from A has the same order of magnitude for A either colocalized with B or remote. In other words, this suggests that gene colocalization is highly favorable for transcription kinetics, but only when it is geometrically-controlled, that is in the case of compact exploration, which makes the experimental characterization of the nature of transport in the nucleus a major issue.

To conclude, we calculated analytically the FPT distribution of a diffusing particle to a target, and showed that transport processes as various as regular diffusion, anomalous diffusion, diffusion in disordered media and in fractals fall into the same universality classes. Our results put forward that geometry, and in particular the initial localization of reactants, can become a key parameter of reaction kinetics in confinement. In particular, this regime of "geometry-controlled kinetics" could be relevant to transcription kinetics and could help understand the crucial role of spatial organization of genes.

\section{Acknowledgments}

Support from ANR grants DYOPTRI and DYNAFT is acknowledged.

\section{Author contributions}

All authors contributed equally to this work.

[1] Rice, S. Diffusion-Limited Reactions (Elsevier, Amsterdam, 1985).

[2] Kopelman, R. Fractal reaction kinetics. Science 241, 1620-1626 (1988).

[3] Shlesinger, M. F., Zaslavsky, G. M. \& Klafter, J. Strange kinetics. Nature 363, 31-37 (1993).

[4] Yu, J., Xiao, J., Ren, X., Lao, K. \& Xie, X. S. Probing gene expression in live cells, one 
protein molecule at a time. Science 311, 1600-1603 (2006).

[5] Elf, J., Li, G.-W. \& Xie, X. S. Probing transcription factor dynamics at the single-molecule level in a living cell. Science 316, 1191-1194 (2007).

[6] Schuss, Z., Singer, A. \& Holcman, D. The narrow escape problem for diffusion in cellular microdomains. Proceedings of the National Academy of Sciences 104, 16098-16103 (2007).

[7] Loverdo, C., Benichou, O., Moreau, M. \& Voituriez, R. Enhanced reaction kinetics in biological cells. Nat Phys 4, 134-137 (2008).

[8] Mirny, L. Biophysics: Cell commuters avoid delays. Nat Phys 4, 93-95 (2008).

[9] Bénichou, O., Loverdo, C., Moreau, M. \& Voituriez, R. Optimizing intermittent reaction paths. Physical Chemistry Chemical Physics 10, 7059-7072 (2008).

[10] Redner, S. A guide to first passage time processes (Cambridge University Press, Cambridge, England, 2001).

[11] Shlesinger, M. F. Mathematical physics: first encounters. Nature 450, 40-41 (2007).

[12] Condamin, S., Benichou, O. \& Moreau, M. First-passage times for random walks in bounded domains. Phys Rev Lett 95, 260601 (2005).

[13] Condamin, S., Benichou, O., Tejedor, V., Voituriez, R. \& Klafter, J. First-passage times in complex scale-invariant media. Nature 450, 77-80 (2007).

[14] Benichou, O. \& Voituriez, R. Narrow-escape time problem: Time needed for a particle to exit a confining domain through a small window. Physical Review Letters 100, 168105-4 (2008).

[15] Benichou, O., Meyer, B., Tejedor, V. \& Voituriez, R. Zero constant formula for first-passage observables in bounded domains. Physical Review Letters 101, 130601-4 (2008).

[16] Bouchaud, J.-P. \& Georges, A. Anomalous diffusion in disordered media: Statistical mechanisms, models and physical applications. Physics Reports 195, 127-293 (1990).

[17] Kurzynski, M., Palacz, K. \& Chelminiak, P. Time course of reactions controlled and gated by intramolecular dynamics of proteins: Predictions of the model of random walk on fractal lattices. Proceedings of the National Academy of Sciences of the United States of America 95, 11685-11690 (1998).

[18] Kolesov, G., Wunderlich, Z., Laikova, O. N., Gelfand, M. S. \& Mirny, L. A. How gene order is influenced by the biophysics of transcription regulation. Proceedings of the National Academy of Sciences 104, 13948-13953 (2007).

[19] Fraser, P. \& Bickmore, W. Nuclear organization of the genome and the potential for gene 
regulation. Nature 447, 413-417 (2007).

[20] Zimmerman, S. B. \& Minton, A. P. Macromolecular crowding: Biochemical, biophysical, and physiological consequences. Annual Review of Biophysics and Biomolecular Structure 22 (1993).

[21] Zhou, H.-X., Rivas, G. \& Minton, A. P. Macromolecular crowding and confinement: Biochemical, biophysical, and potential physiological consequences*. Annual Review of Biophysics 37, 375-397 (2008).

[22] D.Ben-Avraham \& S.Havlin. Diffusion and reactions in fractals and disordered systems (Cambridge University Press, 2000).

[23] de Gennes, P. G. Kinetics of diffusion-controlled processes in dense polymer systems. i. nonentangled regimes. The Journal of Chemical Physics 76, 3316-3321 (1982).

[24] Condamin, S., Benichou, O. \& Moreau, M. Random walks and brownian motion: a method of computation for first-passage times and related quantities in confined geometries. Phys Rev E Stat Nonlin Soft Matter Phys 75, 021111 (2007).

[25] O'Shaughnessy, B. \& Procaccia, I. Diffusion on fractals. Physical Review A 32 (1985).

[26] Montroll, E. W. Random walks on lattices. iii. calculation of first-passage times with application to exciton trapping on photosynthetic units. Journal of Mathematical Physics 10, 753-765 (1969).

[27] Kozak, J. J. \& Balakrishnan, V. Analytic expression for the mean time to absorption for a random walker on the sierpinski gasket. Physical Review E 65 (2002).

[28] Agliari, E. Exact mean first-passage time on the t-graph. Physical Review E (Statistical, Nonlinear, and Soft Matter Physics) 77, 011128-6 (2008).

[29] Haynes, C. P. \& Roberts, A. P. Global first-passage times of fractal lattices. Physical Review E (Statistical, Nonlinear, and Soft Matter Physics) 78, 041111-9 (2008).

[30] Tejedor, V., Bénichou, O. \& Voituriez, R. Global mean first-passage times of random walks on complex networks. Physical Review E 80 (2009).

[31] R.Metzler \& J.Klafter. The random walk's guide to anomalous diffusion: a fractionnal dynamics approach. Phys. Rep. 339, 1-77 (2000).

[32] Wachsmuth, M., Waldeck, W. \& Langowski, J. Anomalous diffusion of fluorescent probes inside living cell nuclei investigated by spatially-resolved fluorescence correlation spectroscopy. Journal Of Molecular Biology 298, 677-689 (2000). 
[33] Golding, E. \& Cox, E. Physical nature of bacterial cytoplasm. Phys. Rev. Lett. 96, 981102 (2006).

[34] Condamin, S., Tejedor, V., Voituriez, R., Benichou, O. \& Klafter, J. Probing microscopic origins of confined subdiffusion by first-passage observables. Proceedings of the National Academy of Sciences 0712158105- (2008).

[35] Montroll, E. W. \& Weiss, G. H. Random walks on lattices. ii. Journal of Mathematical Physics 6, 167-181 (1965).

[36] Shlesinger, M. F. Asymptotic solutions of continuous-time random walks. Journal of Statistical Physics 10, 421-434 (1974).

[37] Condamin, S., Benichou, O. \& Klafter, J. First-passage time distributions for subdiffusion in confined geometry. Phys Rev Lett 98, 250602 (2007).

[38] Parson, R. P. \& Kopelman, R. Percolative versus homogenous energy transport kinetics: time-resolved donor and acceptor fluoresence of isotopic mixed naphthalene crystals. Chemical Physics Letters 87, 528-532 (1982).

[39] Saxton, M. J. A biological interpretation of transient anomalous subdiffusion. ii. reaction kinetics 94, 760-771 (2008).

[40] Malchus, N. \& Weiss, M. Elucidating anomalous protein diffusion in living cells with fluorescence correlation spectroscopy_facts and pitfalls. Journal of Fluorescence (2009).

[41] Monson, E. \& Kopelman, R. Observation of laser speckle effects and nonclassical kinetics in an elementary chemical reaction. Physical Review Letters 85 (2000).

[42] Markstein, M., Markstein, P., Markstein, V. \& Levine, M. S. Genome-wide analysis of clustered dorsal binding sites identifies putative target genes in the drosophila embryo. Proceedings of the National Academy of Sciences of the United States of America 99, 763-768 (2002).

[43] Platani, M., Goldberg, I., Lamond, A. I. \& Swedlow, J. R. Cajal body dynamics and association with chromatin are atp-dependent. Nat Cell Biol 4, 502-508 (2002).

[44] Bancaud, A. et al. Molecular crowding affects diffusion and binding of nuclear proteins in heterochromatin and reveals the fractal organization of chromatin. EMBO J 28, 3785-3798 (2009).

[45] Lebedev, D. V. et al. Fractal nature of chromatin organization in interphase chicken erythrocyte nuclei: Dna structure exhibits biphasic fractal properties. FEBS letters 579, 1465-1468 (2005). 
[46] Lebedev, D. et al. Structural hierarchy of chromatin in chicken erythrocyte nuclei based on small-angle neutron scattering: Fractal nature of the large-scale chromatin organization. Crystallography Reports 53, 110-115 (2008). 


\section{Figure captions :}

Fig 1 : First-passage time distribution (FPT) and geometry controlled kinetics. Is (or is not) the initial position of the particle an important parameter of the kinetics ? We show quantitatively that in the case of compact exploration (eg for dilute solutions), the kinetics turns out to be widely independent of the starting point $\left(S_{1}\right.$ or $\left.S_{2}\right)$, whereas in the non compact exploration case (eg for crowded environments), the position of the starting point strongly influences the search time of the target, leading to "geometry controlled kinetics". This result in particular implies that the kinetics of activation of a gene $T$ by a transcription factor $(\mathrm{TF})$ can be orders of magnitudes faster if the TF is released from a site $S \equiv S_{2}$ which is colocalized with (i.e. in the vicinity of) $T$, as compared to the case where the TF is released from a remote site $S \equiv S_{1}$.

Fig 2: Universal FPT distribution in the non compact and marginal cases. The simulated distribution $G_{T S}(\theta)$ divided by the weight $\Pi_{T S}$ is plotted against the universal theoretical prediction $\psi(\theta) \mathrm{Eq}(5)$. The collapse of various examples onto a single master curve shows the universality of the result. a All non compact and marginal cases (plotted independently in $\mathbf{b}, \mathbf{c}, \mathbf{d}, \mathbf{e})$, collapse onto a single exponential master curve. b: Regular diffusion on a 3D cubic lattice and 2D square lattice for various rectangular domains (of sizes $L_{1} \times L_{2} \times L_{3}$ and $\left.L_{1} \times L_{2}\right)$ and source-target pairs $S(x, y, z)$ and $T(x, y, z)$ whose rectangular coordinates are indicated in the legend inset. Here $\theta$ and $\Pi_{T S}$ are calculated using exact results for $\left\langle\mathbf{T}_{T S}\right\rangle$ and $\overline{\langle\mathbf{T}}_{T}$ given in $[24]$. c, d, e: examples of disordered systems. Here, $\left\langle\mathbf{T}_{T S}\right\rangle$ and $\overline{\langle\mathbf{T}}_{T}$ are evaluated numerically. $\mathbf{c}$ : Diffusion on a 3D percolation cluster above criticality embedded in a $30 \times 30 \times 30$ rectangular domain with link probability $p=0.4$. d: $3 \mathrm{D}$ random barrier model (see text) embedded in a $30 \times 30 \times 30$ rectangular domain. e: $3 \mathrm{D}$ random trap model (see text) embedded in various rectangular domains.

Fig 3 : Universal FPT distributions in the compact case. The simulated distribution $G_{T S}(\theta)$ divided by the weight $\Pi_{T S}$ is plotted against the universal theoretical prediction $\psi(\theta), \operatorname{Eq}(6)$. The collapse for different system sizes $N$ shows the universality of the results. Examples of deterministic fractals: a Diffusion on a Sierpinski gasket (with a target at the apex site) and $\mathbf{b}$ on a $\mathrm{T}$ graph (with a target at the center). Here, exact expressions are used for calculating $\left\langle\mathbf{T}_{T S}\right\rangle$ and $\overline{\langle\mathbf{T}}_{T}$. The insert shows that the scaling function $\psi$ weakly depends on the dimensions $d_{f}$ and $d_{w}$. c Diffusion on a 3D critical percolation cluster (random fractal) embedded in rectangular domains of sizes $\left(L_{1} \times L_{2} \times L_{3}\right)$, as indicated in the 
inset. Here, $\left\langle\mathbf{T}_{T S}\right\rangle$ and $\overline{\langle\mathbf{T}\rangle_{T}}$ are evaluated numerically, and average over pairs of points is performed, to fulfill the scale-invariance hypothesis of the propagator (see text before Eq. (5)).

Fig 4 : Reaction kinetics as quantified by the survival probability $S(t)$, plotted for different source-target distances $r$. The non compact case $\mathbf{a}$ is exemplified by a 3D regular diffusion and the compact case $\mathbf{b}$ by a diffusion on a Sierpinski gasket (with a target at the apex). The theoretical prediction for $S(t)$ is obtained from Eqs.(5)-(6). Here $\theta$ and $\Pi_{T S}$ are calculated using exact results for $\left\langle\mathbf{T}_{T S}\right\rangle$ and $\overline{\langle\mathbf{T}}_{T}$. Quantitatively, the typical reaction time $t_{\text {typ }}$ can be defined, for example, through the median $S\left(t_{\text {typ }}\right)=1 / 2$, indicated by the dotted line. In the non compact case, $t_{\text {typ }}$ weakly depends on the initial position of the reactant, which therefore is not an important parameter of the kinetics. On the contrary, in the compact case, $t_{\text {typ }}$ runs over several orders of magnitude depending on the initial position which, in turn, controls the kinetics. 


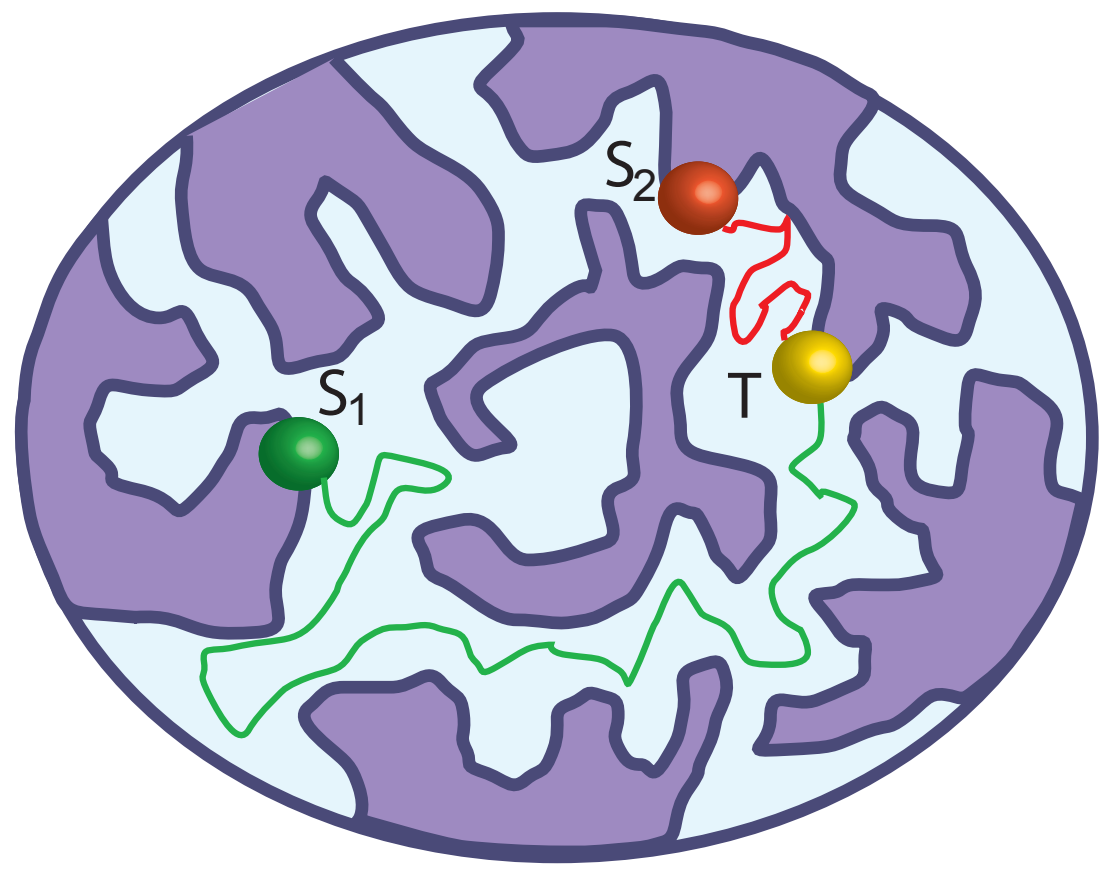

FIG. 1: 

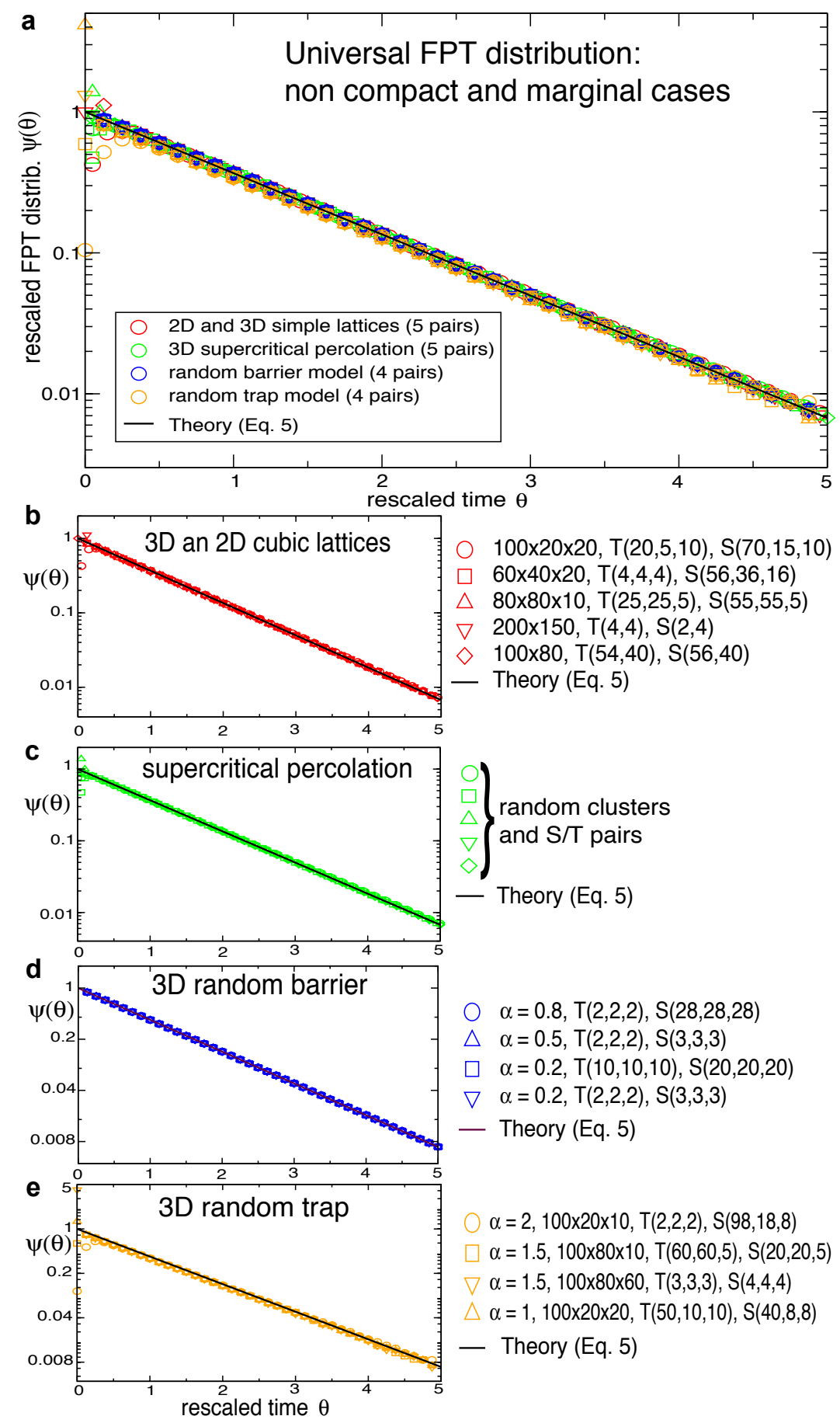

O $\alpha=0.8, T(2,2,2), S(28,28,28)$

$\triangle \alpha=0.5, \mathrm{~T}(2,2,2), \mathrm{S}(3,3,3)$

$\square \alpha=0.2, T(10,10,10), S(20,20,20)$

$\nabla \quad \alpha=0.2, \mathrm{~T}(2,2,2), \mathrm{S}(3,3,3)$

— Theory (Eq. 5)

$\alpha=2,100 \times 20 \times 10, T(2,2,2), S(98,18,8)$

$\square \alpha=1.5,100 \times 80 \times 10, T(60,60,5), S(20,20,5)$

$\nabla \alpha=1.5,100 \times 80 \times 60, T(3,3,3), S(4,4,4)$

$\triangle \alpha=1,100 \times 20 \times 20, \mathrm{~T}(50,10,10), \mathrm{S}(40,8,8)$

- Theory (Eq. 5)

FIG. 2: 

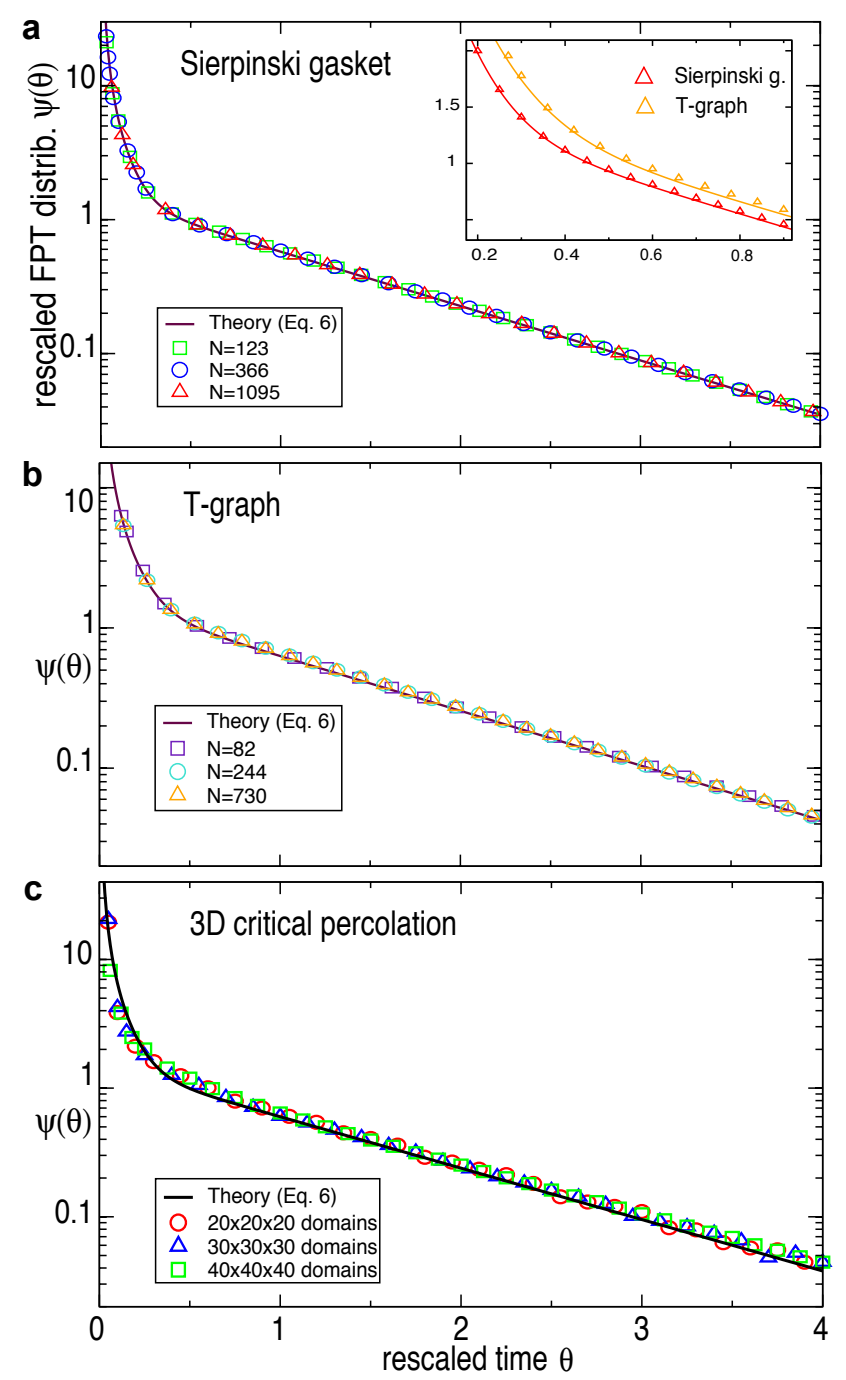

FIG. 3: 

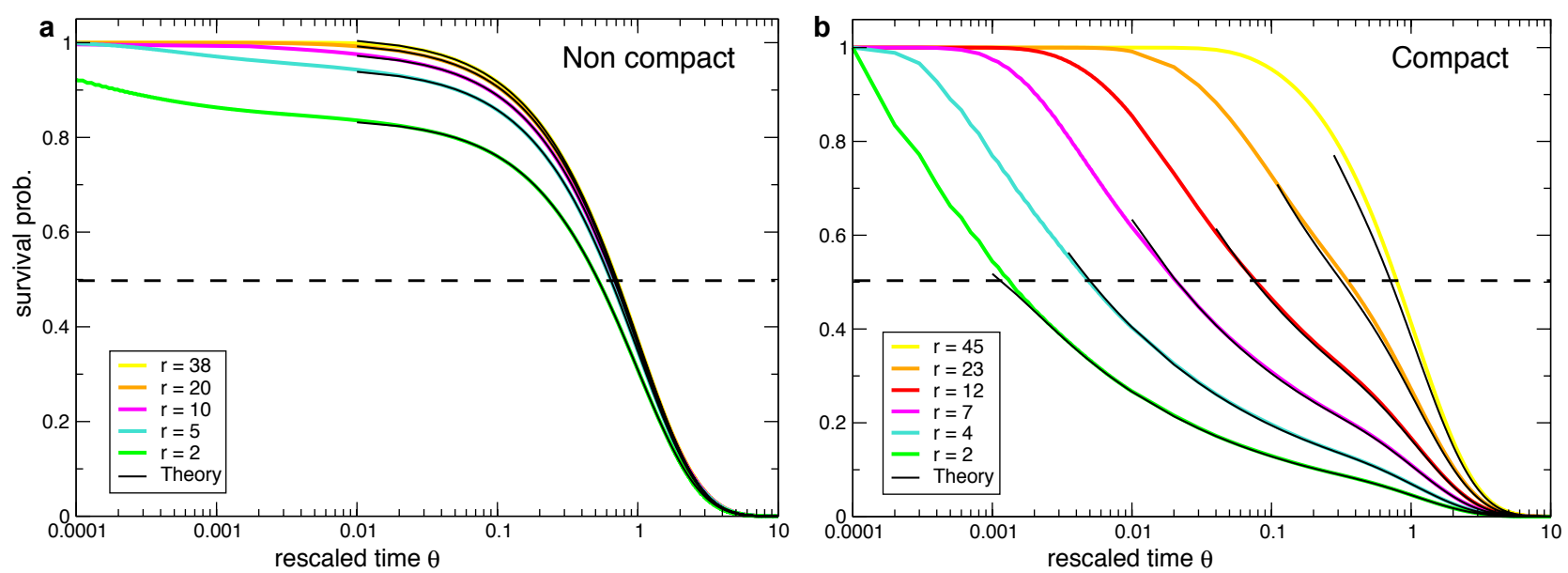

FIG. 4: 